\title{
Power Supply Quality Analysis Using S-Transform and SVM Classifier
}

\author{
Jiaqi Li1, M. V. Chilukuri² \\ ${ }^{1}$ University of Nottingham, Nottingham, UK \\ ${ }^{2}$ SMIEEE, University of Nottingham, Semenyih, Malaysia \\ Email: guildlijiaqi@gmail.com
}

Received January 2014

\begin{abstract}
In this paper, a SVM classifier based on S-Transform is presented for power quality disturbances classification. Firstly, seven types of $P Q$ events are created using Matlab simulation. These signals are analyzed to detect and localize $P Q$ events via $S$-Transform by visual inspection. Then five significant features of the $P Q$ disturbances are extracted from the S-Transform output. Afterwards, $P Q$ disturbance samples with the five features are fed to SVM for training and automatic classification. Besides, particle swarm optimization is implemented to improve the performance of SVM. The results of the classification indicate that SVM classifier is an effective mechanism to detect and classify power quality disturbances.
\end{abstract}

\section{Keywords}

\section{Power Quality Disturbance; S-Transform; SVM}

\section{Introduction}

Nowadays, the electronic equipment and converting technology with high power quality demand are widely applied. Therefore, increasing the electrical power quality has become a significant issue. There are 6 major PQ distortions namely voltage sags, swells, interruption, flicker, oscillatory transients and harmonics. To enhance the electrical power quality, the sources and courses of PQ disturbances have to be detected and classified. Normally, fuzzy expert system cluster classifier and SVM are applied. Recently, these classification technologies include pattern recognition, data mining and order making. Raw data is pre-processed and features are extracted to analyze the received data for automatic PQ problems resolution [1].

Traditionally, it is popular to analyze stationary signal with the Fourier Transform (FT) based method, which loses time the time imagination. In order to achieve effective extraction of information for non-stationary signals, Short Time Fourier Transform (STFT) depicting time-frequency domain issued. However, STFT can partly solve the problem because of the Heisenberg uncertainty principle [2], which makes it hard to detect short time resolution and high frequency resolution. Consequently, another analytical method called Wavelet Transform (WT) [3] is found to have better time-frequency characterization. It provides good frequency resolution for lowfrequency events while offer nice time resolution for high-frequency events. In addition, WT is able to furnish the feature extraction of signals with continuous wavelet transform (CWT) and discrete wavelet transform 
(DWT) [4]. However, wavelet transform is sensitive to noise. In 1996, Stockwell proposed a better approach called S-Transform (ST), which has a hybrid property of STFT and WT. Not only being immune to noise, but also ST utilizes a variable window length and preserves phase information during the decomposition with the application of the FT kernel [5].

Therefore, this paper reveals a group of optimal feature vectors extracted from ST for training the support vector machine (SVM). Then the trained SVM is enabled to classify different PQ disturbances. It should be noticed that the accuracy of the support vector machine is affected by the chosen features and the amount of training data. The PQ disturbance data are simulated using Matlab GUI. Besides, the accuracy of SVM can also be improved by Particle Swarm Optimization.

\section{S-Transform}

S-Transform can be presented in many methods. In this paper, ST is described as continuous wavelet transform (CWT) with Gaussian Window proportional to frequency.

CWT of a function $h(t)$ as:

$$
W(\tau, d)=\int_{-\infty}^{+\infty} h(t) w(d, t-\tau) d t
$$

where $d$ is the scale parameter inverted from the fundamental frequency $f$ defining the width of the wavelet $w(d, t)$. The S-Transform of $h(t)$ can be obtained by multiplying a CWT, including a particular mother wavelet, with the phase factor

besides, the mother wavelet is

$$
S(\tau, f)=e^{-i 2 \pi f t} W(d, \tau)
$$

$$
w(t, f)=\frac{|f|}{\sqrt{2 \pi}} e^{-\frac{t^{2} f^{2}}{2}} e^{-i 2 \pi f t}
$$

The Equation (2) is not a strict CWT because of the failure of satisfying the value of zero mean for a wavelet shown in Equation (3). In the following, the ST can be defined as

$$
S(\tau, f)=\int_{-\infty}^{\infty} h(t)\left(\frac{|f|}{\sqrt{2 \pi}} e^{-\frac{(\tau-t)^{2} f^{2}}{2}}\right) e^{-i 2 \pi f t} d t
$$

and the width of the Gaussian window is defined as

$$
\sigma(f)=T=\frac{1}{|f|}
$$

with Fourier spectrum $H(f)$ of $h(t)$, the ST can be expressed as

$$
S(\tau, f)=\int_{-\infty}^{\infty} H(\alpha+f) e^{-\frac{2 \pi^{2} a^{2}}{f^{2}}} e^{-i 2 \alpha f t} d \alpha, f \neq 0
$$

In the discrete expression, the disturbance signal $h(t)$ is written as $h(k t), k=0,1,2, \ldots N-1$ with a sampling time space $\mathrm{T}$ and a total sampling number N. Its discrete FT can be presented as

$$
H\left[\frac{n}{N T}\right]=\frac{1}{N} \sum_{k=1}^{N-1} h(k T) e^{-\frac{i(2 \pi n k)}{N}}
$$

with $n=0,1,2, \ldots N-1$.

From the Equation (6), the S-Transform of a discrete time series can be achieved via making $\tau$ and $f$ to be $j T$ and $\frac{n}{N T}$ respectively.

$$
S\left[j T, \frac{n}{N T}\right]=\sum_{m=0}^{N-1} H\left[\frac{m+n}{N T}\right] G(m, n) e^{\frac{i 2 \pi m j}{N}}, n \neq 0
$$

where

$$
G(m, n)=e^{-\frac{2 \pi^{2} m^{2}}{b^{2} n^{2}}}
$$


$1 / b, n, m, j=0,1,2, \ldots N-1$, and $N$ is the sampling number. When $n=0$,

$$
S[k T, 0]=\frac{1}{N} \sum_{m=0}^{N-1} H\left[\frac{m}{N T}\right]
$$

therefore, the discrete inverse of $S$-Transform can be achieved

$$
h(k T)=\frac{1}{N} \sum_{n=0}^{N-1}\left\{\sum_{j=0}^{N-1} S\left[\frac{n}{N T}, j T\right]\right\} e^{\frac{i 2 \pi n k}{N}}
$$

The application of the FFT and the convolution theorem makes the discrete ST a quick way of computation.

\section{Pq Analysis Using St}

In this section, six types of power quality disturbances, involving sag, swell, interruption, flicker, oscillatory transient and harmonic, are analyzed. It is not easy to obtain these complex PQ disturbances from utility power quality monitoring system due to confidentiality, thus above signals are simulated using MATLAB GUI. The results of power quality signals simulated are shown from Figures 1-6. In each figure, the first plot display the original waveform of a signal that amplitude versus time. The second axes contours the time-frequency plot revealing normalized frequency versus time ST-amplitude. In the following plot c, the time-maximum amplitude plot displaying maximum amplitude versus time can be achieved by seeking columns of ST-amplitude at every frequency. Besides, it demonstrates the ST-amplitude at fundamental frequency. In the fourth graph, it plots the frequency - maximum amplitude. In this plot, rows of ST amplitude are sought at every frequency to display maximum amplitude versus normalized frequency.

The simulated power quality signals are displayed in the first part of Figures 1-6. In the second part, the outcomes of S-transform matrix are shown in contour plot. Besides, the maximum amplitude of each column and row in the S-transform matrix are illustrated in the third graph and the graph of Figures 1-6 respectively.
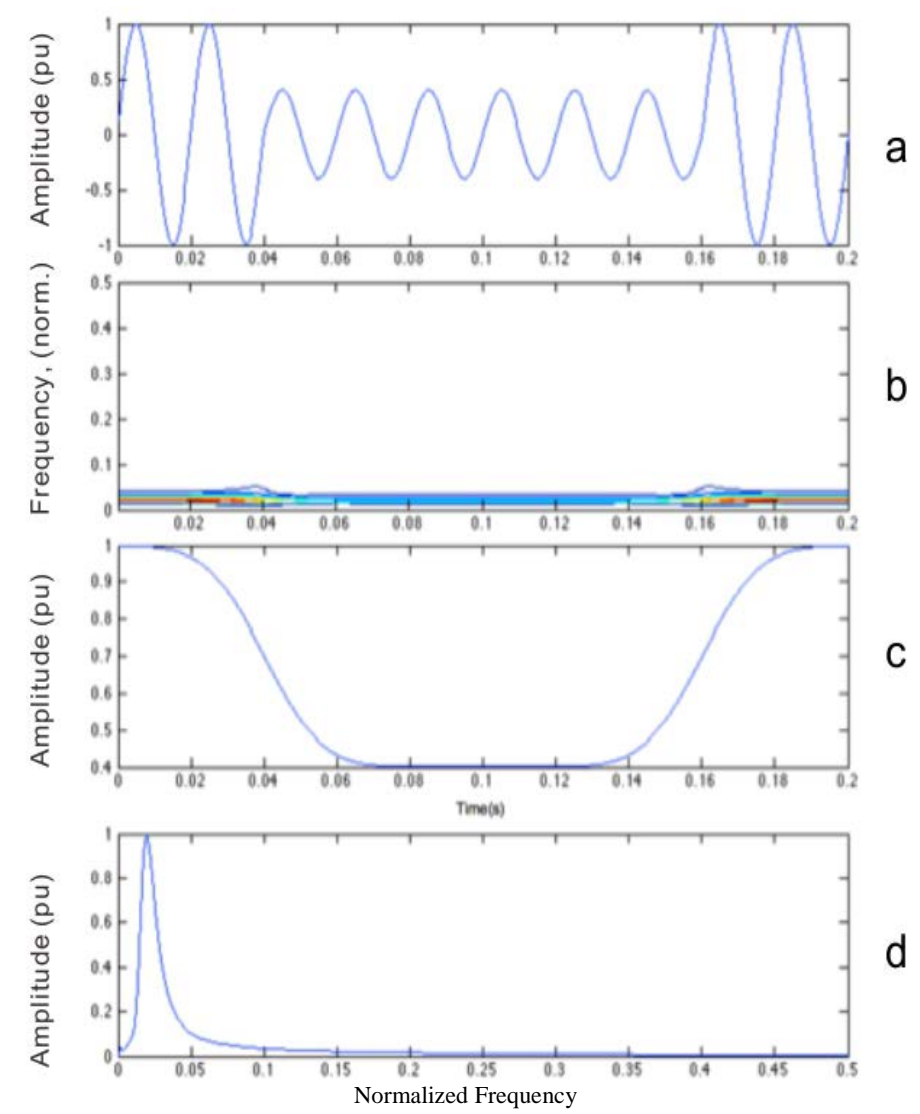

Figure 1. PQ disturbance of sag. 

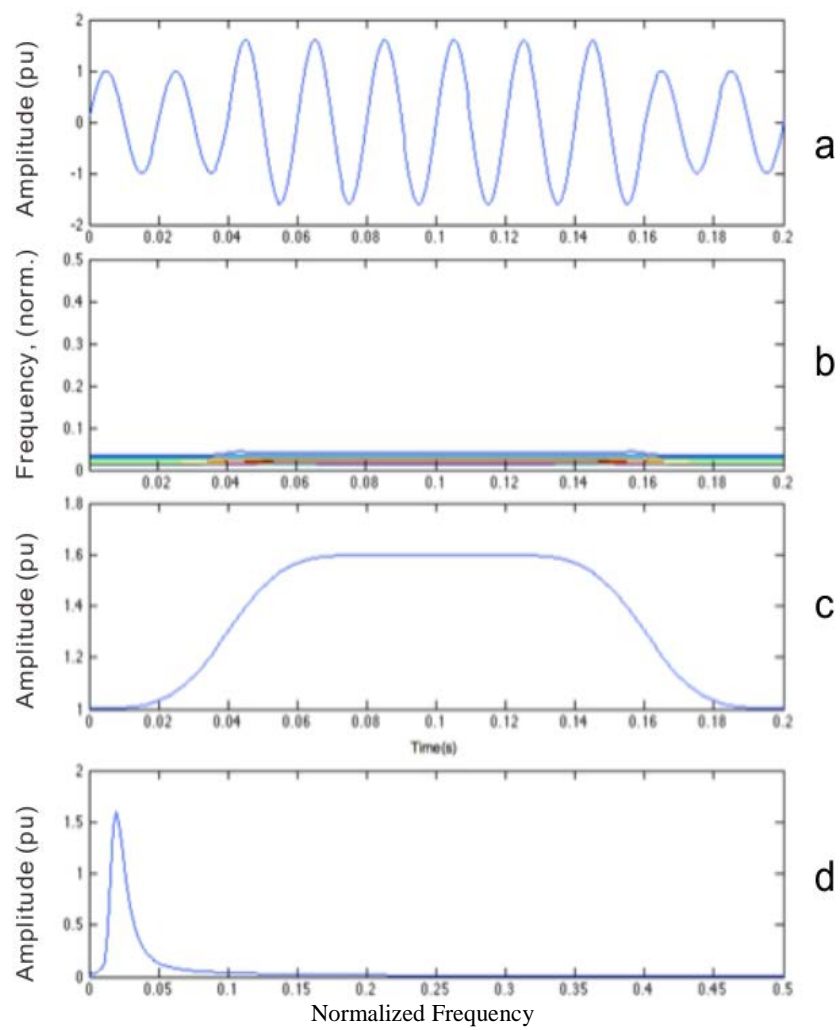

Figure 2. PQ disturbance of swell.
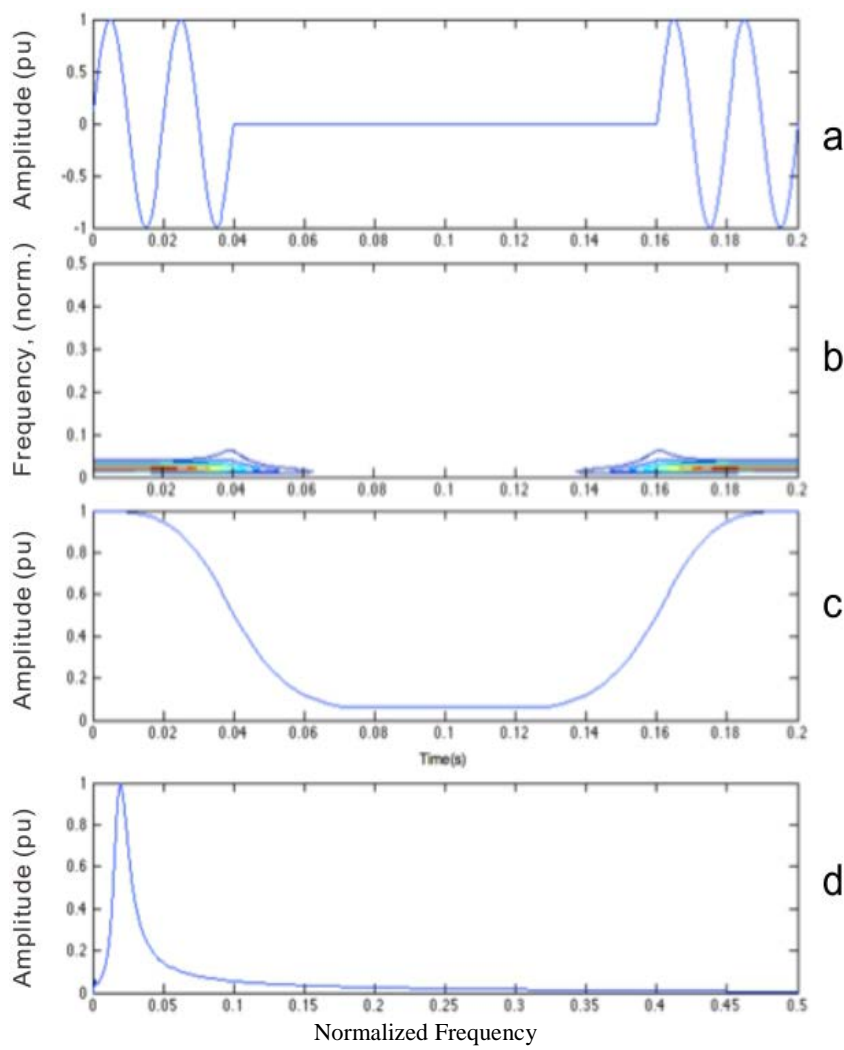

Figure 3. PQ disturbance of interruption. 

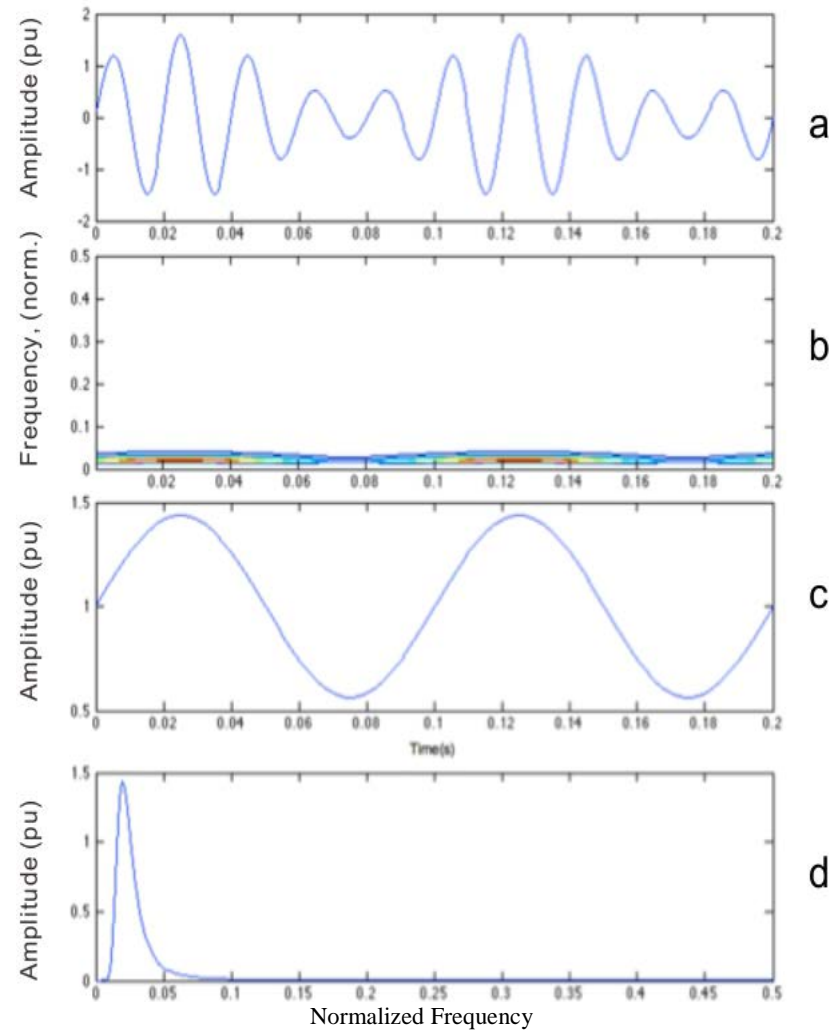

Figure 4. PQ disturbance of flicker.

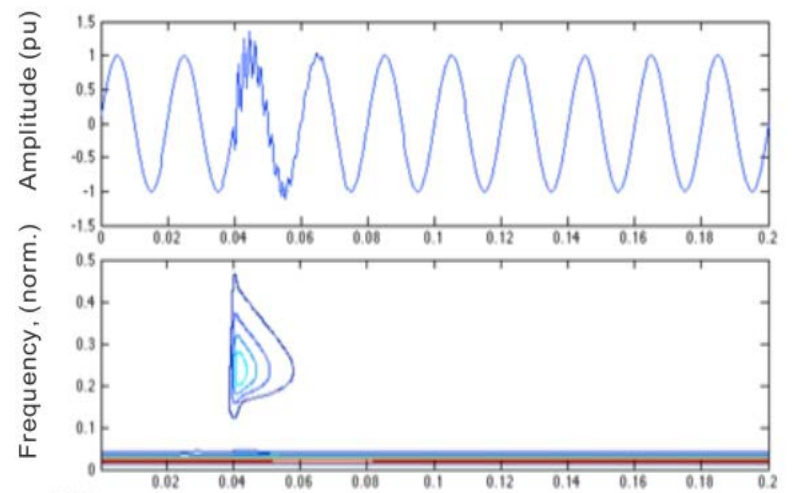

a
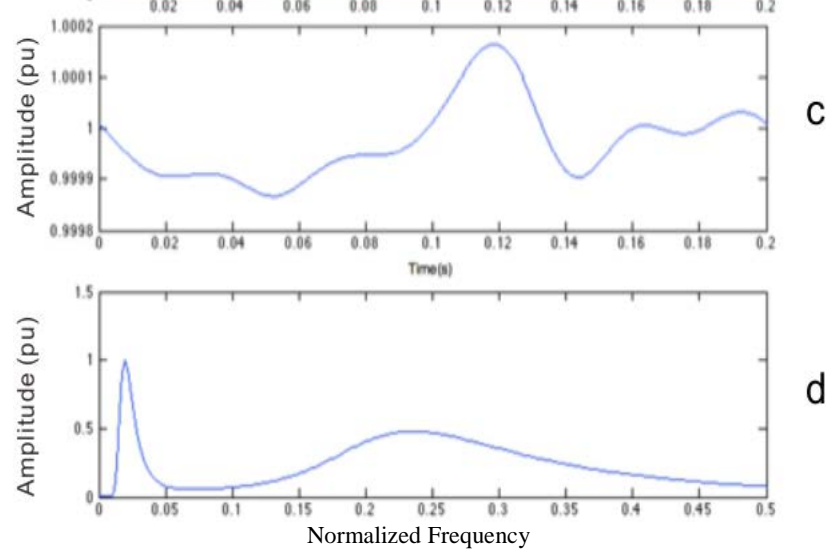

Figure 5. Oscillatory transient. 


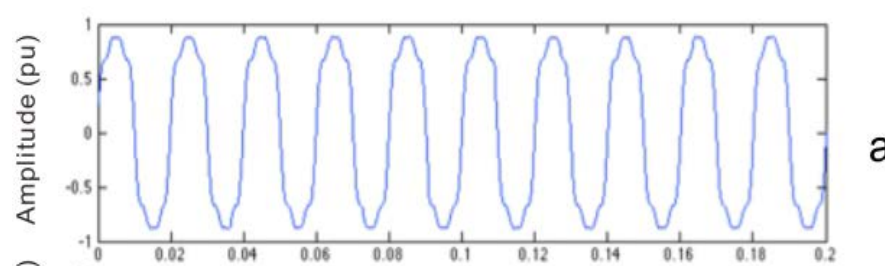

a

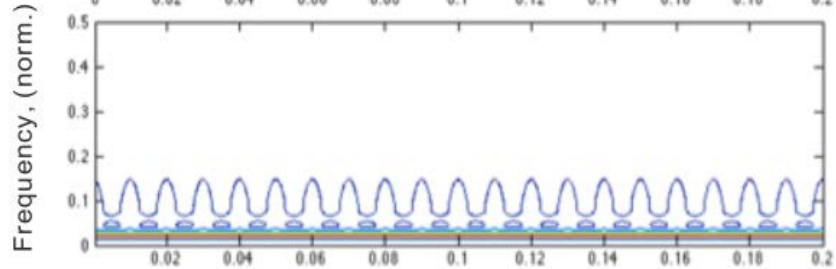

b

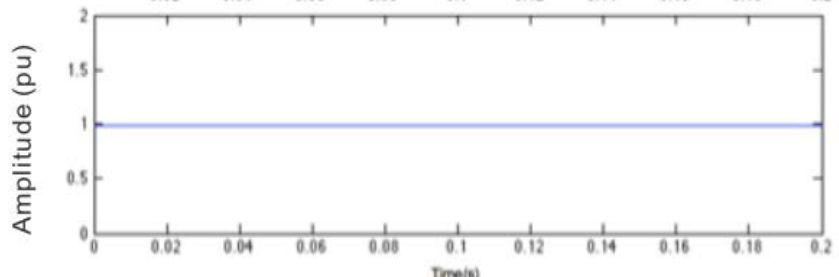

C

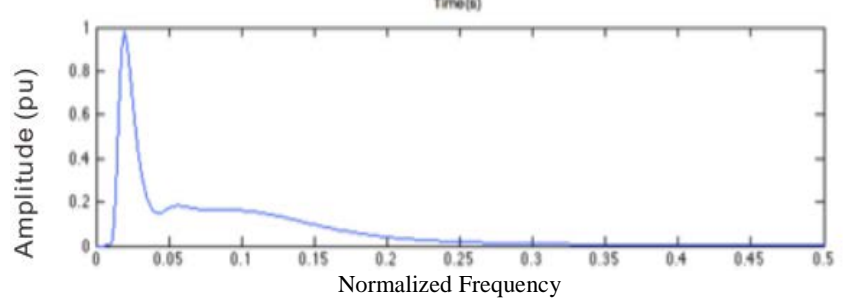

d

Figure 6. PQ disturbance of harmonic.

\section{Features Extraction}

From the PQD plots shown above, major features of power quality disturbance can be extracted. According to the characteristics of seven PQ events, which are normal signal, voltage sag, swell, interruption, flicker, oscillatory transient and harmonic, the following five significant features are computed to classify disturbances.

(a) $F_{1}$ refers to the standard deviation of the time-maximum amplitude plot (TmA-plot) presenting the amplitude of fundamental frequency.

(b) $F_{2}$ is the maximum value of frequency in the time-frequency plot.

(c) $F_{3}$ refers to the frequency-maximum amplitude plot (FmA-plot).

(d) $F_{4}$ is the mean value of TmA-plot.

(e) $F_{5}$ distinguishes voltage sag and interruption by searching the number of peaks in the time-frequency plot.

To demonstrate the relation between the features defined above, 45 sets data of each kind of the seven power quality cases are sampled at a sampling frequency of $2.56 \mathrm{kHz}$. Besides, random white noises with SNR (signal to ratio) rating from 50 to 20 and zero mean is added to these PQ events [6]. For instance, two results are displayed in Figures 7 and 8.

In Figure 7, it presents the seven PQ cases in the plot of Feature 1 versus Feature 2. The magnitudes of disturbances and noises added to the cases are changing for displaying the difference in the feature scatter. From this graph, normal signal, oscillatory and harmonic are clearly separated from the other four power quality disturbances.

Figure 8 shows that voltage swell, flicker, sag and interruption are classified. The black crowd is a group of oscillatory transient, normal signal and harmonic, which does not interfering the classification for the front four disturbances. It proves that feature4 is able to differentiate voltage swell, flicker, sag and interruption effectively.

From these graphs, it is easy to be observed that different types of power quality cases showing varying features can be classified automatically. 


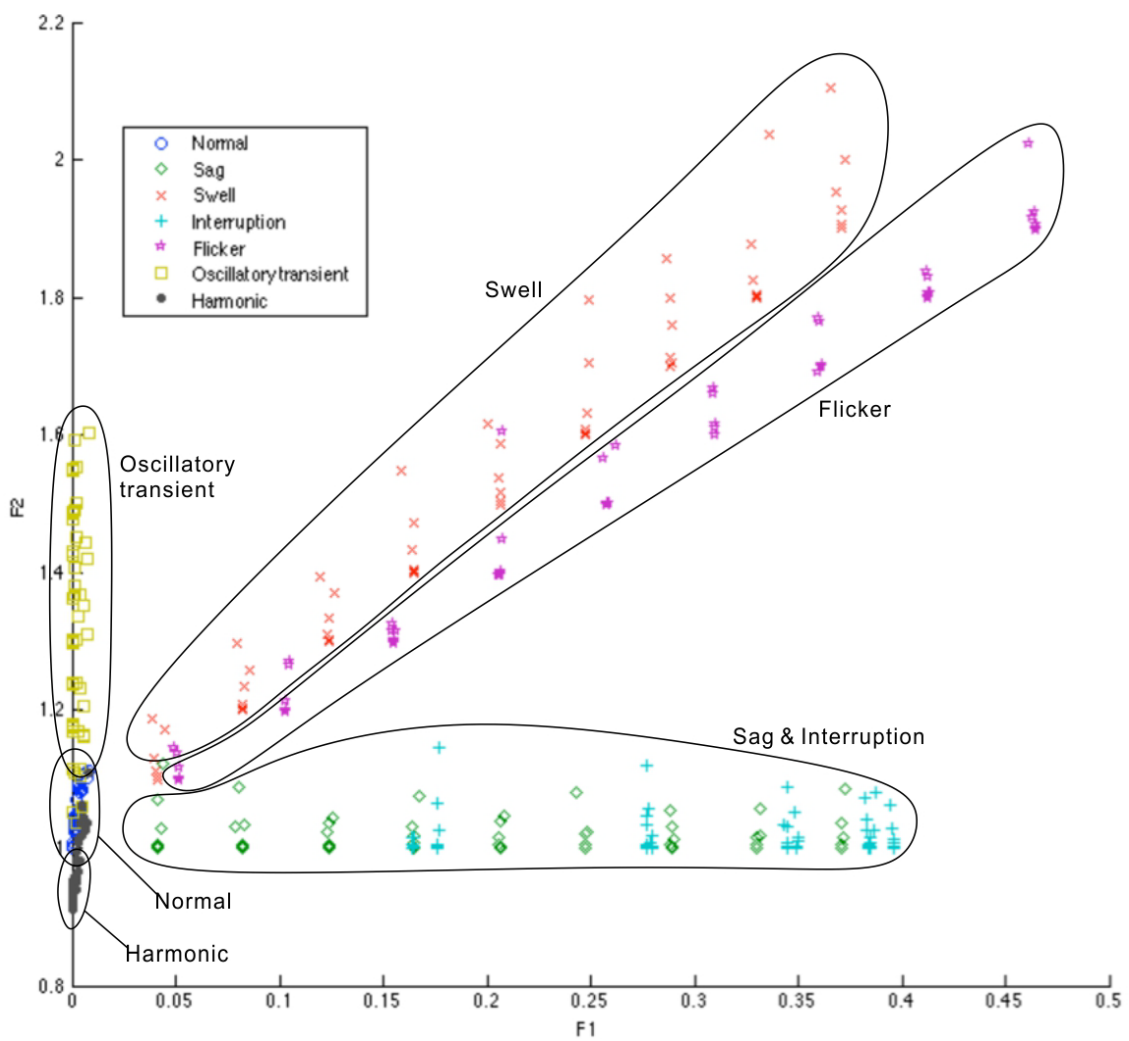

Figure 7. Feature 1 versus Feature 2 scatter.

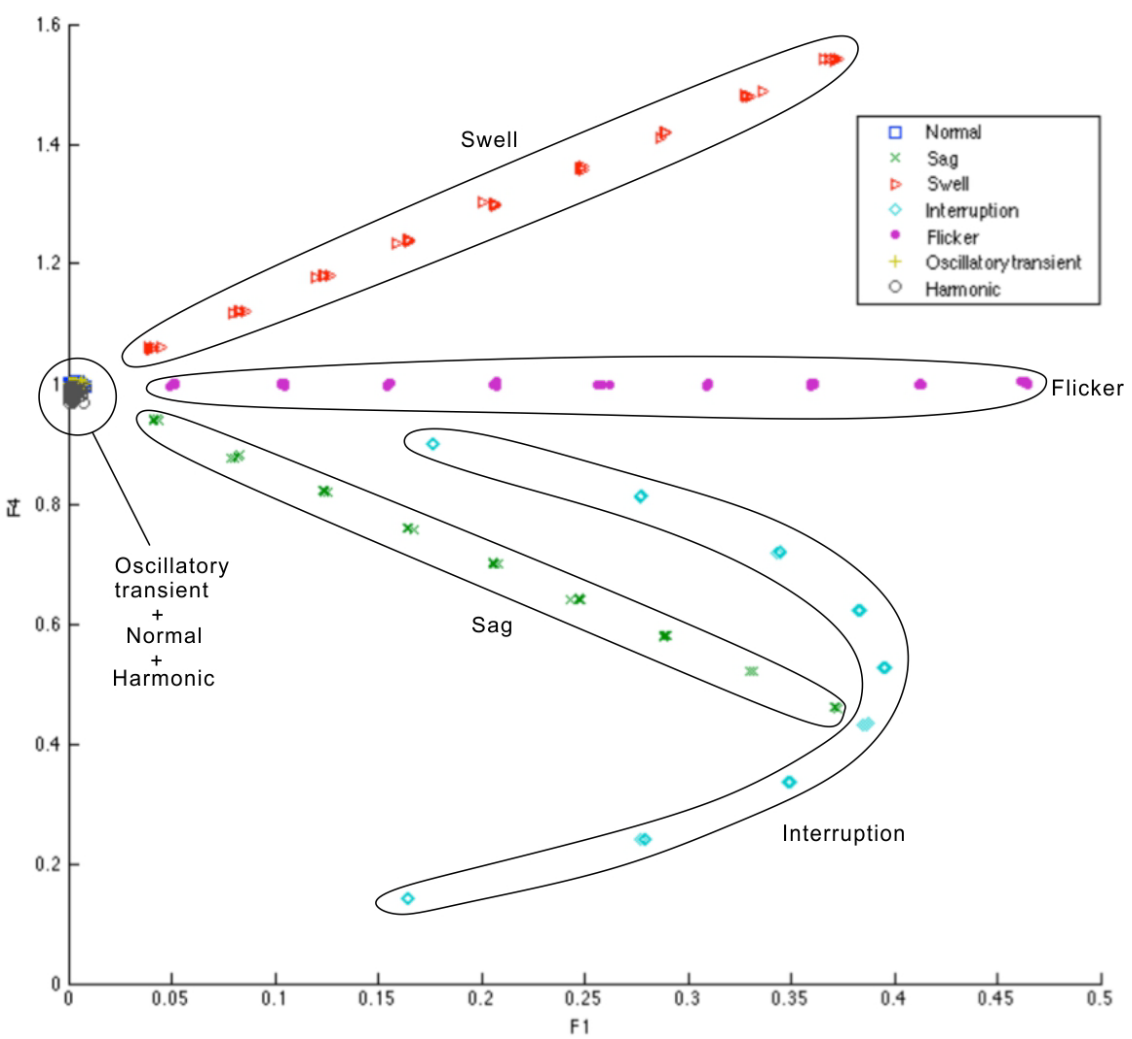

Figure 8. Feature 1 versus Feature 4 plot. 
$F_{1}=0.01$ is a good evidence to distinguish between the group of normal voltage signal, oscillatory transient and harmonic with the group of voltage sag, swell, interruption and flicker. $F_{2}=1.02$ separates harmonic and oscillatory transient while $F_{3}=1.02$ can differentiate normal voltage signal and oscillatory transient in most cases. $F_{4}$ is a strong distinguishing base. For voltage sag and interruption, $F_{4}$ should be smaller than 0.96 . Besides, $F_{4}$ approximately equals to 1 for flicker whereas $F_{4}$ is bigger than 1.05 for voltage swell. Therefore, the following rule base is defined corresponding to different type of power quality disturbances.

\section{Power Quality Disturbance Classification Using Support Vector Machine}

Support vector machine (SVM) is an algorithm with great advantages for classification developed from Statistical Learning Theory by Vapnik [7]. Firstly, it provides accurate classifier with support vectors, which is robust to noise. Secondly, the application of kernel function facilitates SVM to solve nonlinear problems without constructing a feature space with explicit high dimension. Thirdly, SVM is capable to be applied for both binary and multiclass classification. The main procedures of SVM classifier are showed as Figure 9. SVM separating two different groups of data by searching a hyperplane provides higher classification accuracy according to F. Qi et al. [8], though there are subordinate mistakes for small sample, nonlinear and high dimensional pattern recognition issues. As a result, SVM classifier is exploited as pattern classifier for power quality disturbance classification.

After the vectors struct is obtained, predictions for new data can be made by the trained SVM. The best solution $\mathrm{G}$ and the tradeoff parameter $\mathrm{C}$ are two significant factors affecting the prediction accuracy of the SVM model. Nevertheless, the parameters $\mathrm{G}$ and $\mathrm{C}$ are varying, which indicates that it is difficult to gain the optimal one. As a result, partial swarm optimal (PSO) is applied to find the desired G and C for support vector machines automatically.

\section{Discussion}

The data of power quality events used in classification are developed using Matlab simulation with a sampling frequency of $2.56 \mathrm{kHz}$. The seven types of PQ data are simulated with SNR $50 \mathrm{~dB}, 25 \mathrm{~dB}$ or no noise. The training data contains 130 samples for each power quality events, which generated with three levels of disturbance magnitude: $0.3,0.6$ and 0.8 . Then, another 50 samples for each disturbance are generated randomly within the defined range for testing.

The total 350 testing samples are classified by three classifier programmed in Matlab, which are classifier basing on rules of Table 1, SVM classifier and PSO-SVM classifier. The results of classification by the three

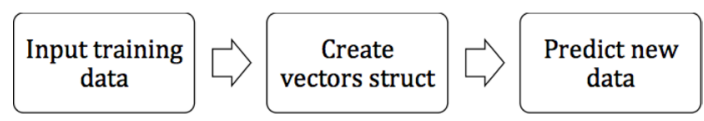

Figure 9. Operating procedures of SVM.

Table 1. Seven rules for classification.

\begin{tabular}{|l|l|}
\hline$R_{1}$ & $\begin{array}{l}\text { If } \quad F_{1}<0.01,1<=F_{2}<=1.2, F_{3}< \\
0.12, \text { then } \mathrm{PQD}=\text { =normal voltage signal; }\end{array}$ \\
\hline$R_{2}$ & $\begin{array}{l}\text { If } F_{1}>0.01, F_{4}<0.96, F_{5}=1, \text { then } \\
\mathrm{PQD}=\text { voltage sag; }\end{array}$ \\
\hline$R_{3}$ & $\begin{array}{l}\text { If } \quad F_{1}>0.01,1.05<F_{4} \quad \text { then } \\
\mathrm{PQD}=\text { voltage swell; }\end{array}$ \\
\hline$R_{4}$ & $\begin{array}{l}\text { If } F_{1}>0.01, F_{4}<0.96, F_{5}=0, \text { then } \\
\mathrm{PQD}=\text { voltage interruption; }\end{array}$ \\
\hline$R_{5}$ & $\begin{array}{l}\text { If } F_{1}>0.01,0.99<F_{4}<1.02, \text { then } \\
\mathrm{PQD}=\text { flicker; }\end{array}$ \\
\hline$R_{6}$ & $\begin{array}{l}\text { If } \quad F_{1}<0.01,1.1<F_{2}, 0.12<F_{3} \quad, \\
\text { then } \mathrm{PQD}=0 \text { oscillatory transient; }\end{array}$ \\
\hline$R_{7}$ & $\begin{array}{l}\text { If } \quad F_{1}<0.01, F_{2}<1.1,0.12<F_{3} \quad, \\
\text { then } \mathrm{PQD}=\text { harmonic. }\end{array}$ \\
\hline
\end{tabular}


classifiers are displayed below.

\section{Conclusion}

The classification results reveal that three classifiers can have a good identification for different power quality cases, though the accuracy reduces by decreasing SNR. The classifier basing on rules can increase the accuracy by modifying the settings of boundaries between seven PQ events. However, the complexity of real power quality events makes it hardly applicable in practice. From Tables 2-4, PSO-SVM is proved to be a better classifier

Table 2. Classification results of PQD without noise.

\begin{tabular}{|c|c|c|c|}
\hline PQ event & $\begin{array}{c}\text { Rule } \\
\text { Classifier }\end{array}$ & SVM & PSOSVM \\
\hline Normal & $50 / 50$ & $50 / 50$ & $50 / 50$ \\
\hline Sag & $50 / 50$ & $49 / 50$ & $49 / 50$ \\
\hline Swell & $50 / 50$ & $50 / 50$ & $50 / 50$ \\
\hline Interruption & $50 / 50$ & $50 / 50$ & $50 / 50$ \\
\hline Flicker & $49 / 50$ & $50 / 50$ & $50 / 50$ \\
\hline $\begin{array}{c}\text { Oscillatory } \\
\text { transient }\end{array}$ & $49 / 50$ & $48 / 50$ & $48 / 50$ \\
\hline Harmonic & $50 / 50$ & $50 / 50$ & $50 / 50$ \\
\hline Total Success & $348 / 350$ & $345 / 350$ & $347 / 350$ \\
\hline
\end{tabular}

Table 3. Classification results of pqd with $50 \mathrm{db}$ snr.

\begin{tabular}{|c|c|c|c|}
\hline PQ event & $\begin{array}{c}\text { Rule } \\
\text { Classifier }\end{array}$ & SVM & PSOSVM \\
\hline Normal & $50 / 50$ & $50 / 50$ & $50 / 50$ \\
\hline Sag & $44 / 50$ & $46 / 50$ & $48 / 50$ \\
\hline Swell & $50 / 50$ & $48 / 50$ & $50 / 50$ \\
\hline Interruption & $46 / 50$ & $49 / 50$ & $47 / 50$ \\
\hline Flicker & $48 / 50$ & $47 / 50$ & $49 / 50$ \\
\hline $\begin{array}{c}\text { Oscillatory } \\
\text { transient }\end{array}$ & $43 / 50$ & $45 / 50$ & $46 / 50$ \\
\hline Harmonic & $49 / 50$ & $48 / 50$ & $48 / 50$ \\
\hline Total Success & $330 / 350$ & $333 / 350$ & $338 / 350$ \\
\hline
\end{tabular}

Table 4. Classification results of pqd with $25 \mathrm{db}$ snr.

\begin{tabular}{|c|c|c|c|}
\hline PQ event & $\begin{array}{c}\text { Rule } \\
\text { Classifier }\end{array}$ & SVM & PSOSVM \\
\hline Normal & $48 / 50$ & $49 / 50$ & $50 / 50$ \\
\hline Sag & $41 / 50$ & $42 / 50$ & $42 / 50$ \\
\hline Swell & $48 / 50$ & $48 / 50$ & $50 / 50$ \\
\hline Interruption & $45 / 50$ & $46 / 50$ & $46 / 50$ \\
\hline Flicker & $45 / 50$ & $46 / 50$ & $48 / 50$ \\
\hline $\begin{array}{c}\text { Oscillatory } \\
\text { transient }\end{array}$ & $40 / 50$ & $42 / 50$ & $44 / 50$ \\
\hline Harmonic & $47 / 50$ & $47 / 50$ & $48 / 50$ \\
\hline Total Success & $314 / 350$ & $321 / 350$ & $332 / 350$ \\
\hline
\end{tabular}


than SVM, which has the highest accuracy and best robust to noise. Therefore, it is great potential for this classifier with on-line classification capability to enhance the performance of automatic power quality monitoring devices.

\section{References}

[1] Flores, R. (2003) Signal Processing Tools for Power Quality Event Classification.

[2] Mallat, S. (1989) A Theory for Multiresolution Signal Decomposition: The Wavelet Representation.

[3] Stockwell, R.G., Mansinha, L. and Lowe, R.P. (1996) Localization of the Complex Spectrum: The S-Transform.

[4] Vapnik, V. (1998) The Support Vector Method of Function Estimation.

[5] Batista, J., Afonso, J.L. and Martins, J.S. (2003) Low-Cost Power Quality Monitor Based on a PC.

[6] Chilukuri, M.V., Dash, P.K. and Basu, K.P. (2004) Time-Frequency Based Pattern Recognition Technique for Detection and Classification of Power Quality Disturbances.

[7] Vapnik, V.N. (1995) The Nature of Statistical Learning Theory. Springer-Verlag, New York. http://dx.doi.org/10.1007/978-1-4757-2440-0

[8] Qi, F., Bao, C. and Liu, Y. (2004) A Novel Two-Step SVM Classifier for Voiced/Unvoiced/Silence Classification of Speech. 